\title{
Demystifying sexual connotations: A model for facilitating the teaching of intimate care to nursing students in South Africa
}

\author{
S Shakwane, $\mathrm{PhD}$; S Mokoboto-Zwane, $\mathrm{PhD}$ \\ College of Human Sciences, Department of Health Studies, University of South Africa, Pretoria, South Africa
}

Corresponding author: S Shakwane (shakws@unisa.ac.za)

Background. The concept of intimate care is not freely used in nursing education and practice. Nursing students provide basic nursing care that requires physical and psychological closeness to diverse patients. During the execution of some basic nursing care, patients' body parts that are considered intimate, private and sexual, are exposed and touched. This encounter with a patient's naked body may bring about feelings of anxiety, embarrassment and discomfort for nursing students and patients.

Objective. To develop and describe a model for facilitating the teaching of intimate care to nursing students in South Africa.

Methods. The study was conducted at two nursing education institutions (NEIs) in Gauteng Province. A qualitative, theory-generating, research design that is explorative, descriptive and contextual in nature was used to develop a model for facilitating the teaching of intimate care to nursing students. A combination of stages of theory-generating approaches was used to identify and define the main concept. The structure and process of the model were visually portrayed and described.

Results. The findings of this study demonstrate that intimate care is not facilitated in NEIs and nursing students are not well prepared and supported to provide intimate care to diverse patients competently, confidently and comfortably. Facilitating the teaching of intimate care to nursing students was identified as the main concept. Based on this, a model to facilitate the teaching of intimate care to nursing students was therefore proposed, described and visually illustrated.

Conclusion. The developed model will assist nurse educators in facilitating the teaching of intimate care in NEIs and in providing continuous support to nursing students. It will empower students to implement intimate care competently, comfortably and confidently, enabling them to establish nurse-patient intimate care relationships based on trust, respect and dignity.

Afr J Health Professions Educ 2020;12(3):103-108. https://doi.org/10.7196/AJHPE.2020.v12i3.1367

Intimate care comprises physical touch that involves inspection of, and physical contact with body parts, which can cause embarrassment to the patient and the nurse. ${ }^{[1]}$ It is further described as task-orientated touch to areas of the patient's body that might create feelings of discomfort, anxiety and fear or might be misinterpreted as having a sexual purpose. ${ }^{[2]}$ Patients seek physical or psychological care at healthcare institutions. Nurses provide care that meets the needs of the patient, and these needs may require a nurse to be physically and psychologically close to a patient. This closeness could include exposure of private body parts, touching of the naked body and sharing of intimate information with a total stranger. When providing physical care, touch is necessary and an essential and intrinsic part of a caring profession, ${ }^{[3]}$ as it facilitates communication between the nurse and the patient. The interactions between a nurse and a patient are classified as intimate because they cross the patient's physical and psychological private zones. ${ }^{[4]}$ During intimate care, a nurse and a patient need to establish a relationship in a confined space, where a patient has to allow a stranger to access his/her body's most intimate parts and, in turn, the nurse has to see and touch a body that is not theirs. ${ }^{[5]}$ Touching the patient's body may initiate feelings of discomfort, ${ }^{[3]}$ because the care involves parts of the body that need to be touched.

There is reluctance in using the concept 'intimate care' in the nursing profession. This is based on the assumption that establishing an intimate care relationship with a patient is a violation of the personal and professional ethical boundaries between nurse and patient. ${ }^{[6]}$ However, in the execution of their duties, nursing students are expected to touch areas of a patient's body that are considered private and emotionally sensitive. Many nurses are young, inexperienced with regard to social maturity and responsibility, and may struggle to take on the professional responsibility of providing intimate care to diverse patients, who are strangers to them. ${ }^{[7]}$ Professional intimate care responsibility is entrusted to students, and they are expected to excel at it. However, little is known about how nurses learn, rehearse and incorporate appropriate touch strategies, and there is no model for the use of intimate care/touch in a non-sexual context. ${ }^{[1,2]}$ Nursing students also receive limited instruction in this regard from nursing education institutions (NEIs). ${ }^{\left[{ }^{[3}\right.}$ Young nurses are rudely awakened to the reality of intimate care work once they are placed in the clinical area. At the onset of training, they do not necessarily understand the degree to which nursing care includes intimate care and the discomfort they may experience in such situations. ${ }^{[7]}$

To add to the complexity of this issue, South Africa (SA) is challenged with increased gender-based violence against women and children. ${ }^{[9]}$ Within this context, men and women choose caring careers, such as nursing. The patriarchal and socially constructed gender roles, which perceive nursing as a female profession, ${ }^{[10]}$ may make it even more difficult for male nursing students to provide professional intimate care or touch. The touch from a man is sexualised and, sometimes, viewed as suspicious. ${ }^{[1]}$ These perspectives are further strengthened by media reports of male paedophiles and sexual offenders. ${ }^{[1]}$ In an attempt to protect nurses, Mauritian nursing uses a gender-segregation approach, where male and female patients 
and nurses are separated. This move was deemed relevant to deal with the sexualisation of touch, which suggests the risk of sexual abuse and molestation by the misuse of position and power. Male nurses were believed to make sexualised advances towards female patients, and male patients were likely to do the same towards female nurses. ${ }^{[12]}$

The Constitution of the Republic of South Africa is based on the values of ubuntu (humaneness). ${ }^{[13]}$ Chapter 2(10) pronounces that human dignity must be respected and protected, and section 12(2) indicates that every person has the right to bodily and psychological integrity. ${ }^{[14]}$ NEIs are responsible for preparing nursing students to provide intimate care, while ensuring that it is done with respect and safeguarding of the dignity and integrity of the patient.

Many scholars ${ }^{[1,7]}$ have focused on the experiences of male nurses when providing intimate care and the strategies used to protect them. Little is known about the experiences of female nursing students. Nurse educators ought to create safe environments that simulate intimate care experiences for male and female students. NEIs must create cultural congruent nursing education, which encourages behaviour and decisions that are dignified and appropriate with regard to cultural values, with meaningful, beneficial and acceptable intimate care provision. ${ }^{[15]}$

\section{Methods}

This article forms part of a larger theory-generative study, using a qualitative research design. The larger research project had two phases: an empirical and a theory-generative phase. This paper presents the latter, which was aimed at developing a model for facilitating the teaching of intimate care to nursing students in SA.

It describes a model to facilitate the teaching of intimate care. The three steps of model development, adapted from Chinn and Kramer, ${ }^{[16]}$ as well as from Walker and Avant, ${ }^{[17]}$ are discussed. The first step is concept clarification, which allows the researcher to identify, classify and define the main concept. The second step is the construction of the relationship statement to create the meaning of the model. The third step is the description of the model structure and process.

\section{Step 1: Concept clarification}

A concept is a mental image of a phenomenon, an idea or a construct about a thing or action. Concepts help in identifying the similarity of experiences that are equivalent by categorising all the things that are alike. ${ }^{[17]}$ The main concepts in this study are identified, classified and defined to ensure clarity, as set out below.

\section{Identification of the main concepts}

Structuring a model requires identification of concepts that will form the basis of the model. A model emerges because of the conviction that the existing knowledge and theories are not adequate to represent a phenomenon. ${ }^{[16]}$ For this study, the concepts were derived from the research findings in the empirical phase.

The analysis of data in the empirical phase revealed that intimate care is not considered a skill that needs to be taught to nursing students. This is evident by its exclusion in the curriculum of NEIs in SA. Nursing students are taught procedural principles of nursing care, but not intimate care or touch. They do not know how and when to prepare a patient for touch. Failure to teach intimate care to nursing students has led to experiences of embarrassment, discomfort, anxiety and fear when executing intimate care and have caused some patients to judge intimate care or touch as sexual or inappropriate. ${ }^{[18]}$ How intimate care or touch is experienced by the nurse or patient is based on their culture, religion, age, gender and sexual orientation. ${ }^{[12]}$ Empirical data also show that nursing students in clinical placement are not given adequate support when experiencing challenges in this regard. Lack of preparation of and support for nursing students with regard to intimate care may adversely affect them emotionally and professionally, ${ }^{[18]}$ and patients may not receive quality care. Therefore, there is an imperative for NEIs to facilitate the teaching of intimate care.

Based on the findings of the empirical study, 'facilitating the teaching of intimate care' was identified as the main concept used to develop a model.

\section{Classification of the main concepts}

The main concept was classified using the survey list in Dickoff et al. ${ }^{[19}$ Table 1 displays the integration of the survey facts into the presented model.

\section{Definition of the main concept}

The main concept was defined using these three steps: the dictionary definition, contextual (subject) definition and exemplary or model case. ${ }^{[16]}$ To clarify the main concept, i.e. facilitating the teaching of intimate care,

Table 1. Intimate care model classification ${ }^{[18]}$

\begin{tabular}{ll}
\hline Agent & Nurse educators \\
\hline Recipients & Nursing students \\
Procedure & NEIs should acknowledge and accept that intimate care is a skill that needs to be taught and learnt by nursing students \\
& Intimate care should be included in the nursing programme or curriculum and be taught to nursing students \\
Dynamic & NEIs' willingness to integrate intimate care as a subject, a clinical objective and procedure in their curriculum \\
& Competency of nurse educators in teaching and simulating intimate care skills \\
& Age, culture, gender and sexual orientation of nursing students, nurse educators and patients \\
& Orientation of professional nurses in clinical facilities in facilitating, supervising and supporting nursing students when providing \\
intimate care &
\end{tabular}


each of the four core words that comprise the concept was defined separately, and their attributes were combined. After exploring the dictionary and contextual definition of each word, a list of essential and related attributes was identified.

The theoretical definition of the concepts in the model is defined in Table 2.

\section{Step 2: Construction of relationship statements of the model}

Relationship statements structurally interrelate the concepts of the model. These statements declare a relationship between two or more concepts. ${ }^{[16]}$

Provision of intimate care requires a nursing student to touch a patient, and it also requires a patient to allow a nursing student, who is a stranger, to touch parts of his/her body that are considered intimate, private and sexual. This care takes place in a confined private space. Nursing students are often not well prepared to provide such care. Therefore, facilitating the teaching of intimate care allows nursing students to gain intimate care knowledge and skills. The central concept suggests that nurse educators need to prepare and support nursing students by facilitating the teaching of intimate care for nurses to provide such care competently, confidently and comfortably.

\section{Step 3: Description of the model}

The context, purpose and assumptions of the model are discussed.

\section{Context of the model}

SA is a diverse democratic country - students from different backgrounds are recruited to the nurse training programme. Nursing students are trained to provide care that is non-discriminatory and culturally sensitive, ${ }^{[28]}$ and maintains the dignity and integrity of a patient. Intimate care and touch have a different meaning to different people, based on their sociocultural construction of the human body and touch. Nursing educators need to facilitate the process of teaching intimate care to nursing students to enable them to be aware of their own cultural and religious values regarding caring for a fragile body of a person who differs from them. Intimate care knowledge and skills must include cultural, religious and ethical teaching regarding the human body and nakedness, and humanistic simulation must be used as an effective intimate care teaching strategy. Simulation can enable and encourage nursing students to provide intimate care in a safe environment and expose them to the simulated reality of a human body during intimate care. Nurse educators also need to provide clinical support to nursing students when they render intimate care to patients in a clinical facility.

Table 2. Theoretical definition of the concepts

\begin{tabular}{|c|c|}
\hline Concept & Definition \\
\hline Facilitating & $\begin{array}{l}\text { An interactive learning process, which is goal orientated and dynamic, and in which learners and educators } \\
\text { work together in an atmosphere of genuine mutual respect }{ }^{[20]} \text { Facilitation takes place in the classroom, clinical } \\
\text { laboratory and clinical facilities }\end{array}$ \\
\hline Teaching & $\begin{array}{l}\text { A mental process that produces beneficial and purposeful student learning through the use of appropriate } \\
\text { procedures }^{[20]} \\
\text { Teaching in nursing education is a task-orientated process, which focuses on skills and knowledge } \\
\text { It aims at producing a skilled, knowledgeable, useful and willing nurse }{ }^{[21]}\end{array}$ \\
\hline Intimate care & $\begin{array}{l}\text { Basic nursing care routines that involve procedures, such as provision or assistance with hygiene and urinary } \\
\text { and faecal elimination } \\
\text { Intimate care involves physical or psychological closeness between a nurse and a patient } \\
\text { The nurse comes into contact with a patient's body and the patient must allow a nurse to touch his or her body } \\
\text { This type of care is viewed as an invasion of personal or private space, because the body parts that are exposed } \\
\text { and touched are considered to be private, sexual or intimate }{ }^{[18]} \\
\text { Feelings of discomfort, embarrassment, fear and anxiety may be experienced by the nurse and patient }{ }^{[18]} \\
\text { The nurse's touch may be misinterpreted as inappropriate or sexual }\end{array}$ \\
\hline Competence & $\begin{array}{l}\text { This refers to the ability of a nurse to integrate cognitive, affective and psychomotor skills when delivering } \\
\text { nursing care }{ }^{[22]} \\
\text { Competency assists in professional and interpersonal decision-making for the practice role in the context of } \\
\text { the nursing profession }{ }^{[23]} \\
\text { It also includes having insight and awareness of one's expertise and limitations }{ }^{[24]}\end{array}$ \\
\hline Confidence & $\begin{array}{l}\text { This is a human quality demonstrated through an efficient act, appearance and performance, resulting in a } \\
\text { positive outcome } \\
\text { The process and outcome of the action are evaluated, subjectively and objectively, by the person and by others } \\
\text { who observe the behaviour }{ }^{[25]}\end{array}$ \\
\hline Comfort & $\begin{array}{l}\text { Kolcaba }{ }^{[26]} \text { defines comfort in nursing as the satisfaction of basic human needs for relief, ease or transcendence } \\
\text { arising from a healthcare situation that is stressful } \\
\text { It means being in a state of physical or mental comfort, content and undisturbed } \\
\text { It also means being easy to associate with and deal with people }\end{array}$ \\
\hline Therapeutic nurse-patient relationship & $\begin{array}{l}\text { This is a planned, time-limited and goal-directed connection between a nurse and a patient } \\
\text { It protects the patient's dignity, autonomy and privacy, allowing for the development of trust and respect }{ }^{[27]} \\
\text { In this model, a therapeutic nurse-patient intimate relationship will mean a mutual intimate relationship } \\
\text { established by a nurse and a patient, based on trust, respect and dignity }\end{array}$ \\
\hline
\end{tabular}




\section{Purpose of the model}

Use of this model is specific to nursing education and clinical practice. It seeks to facilitate the teaching of intimate care to nursing students in SA, thereby ensuring that the following objectives are achieved:

- The facilitation of teaching of intimate care at NEIs and ensuring that teaching of intimate care is facilitated by using simulation as an effective teaching method for intimate care provision.

- The adoption of the proposed model for intimate care instruction as a framework for nurse educators when teaching intimate care knowledge and skills to nursing students. The latter will be more empowered to provide intimate care competently, comfortably and confidently, regardless of gender, age, religion, culture and sexual orientation.

- The model provides practical guidelines to prepare nurse educators for their role as facilitators of intimate care.

\section{Assumptions of the model}

The assumptions are the basic givens or accepted truths that are fundamental to theoretical reasoning. They are structural components of a theory that is taken for granted or thought to be true without systematically generated empirical evidence. They are assumed to be true within the model, because they are reasonable. ${ }^{[16]}$ The assumptions of this model are:

- Nurse-patient interaction occurs during the provision of basic nursing care to diverse patients. Most basic nursing care procedures are intimate, as they require physical closeness and touching of a patient.

- Nursing students are not well prepared or trained to provide intimate care to diverse patients competently, comfortably and confidently. During the implementation of intimate care, the patient's private space is invaded, either physically or psychologically. This care can awaken feelings of embarrassment, anxiety, fear and discomfort for nursing students and patients alike. Patients can sometimes misinterpret the nursing students' touch as sexual or inappropriate. NEIs and clinical facilities do not provide sufficient support to nursing students when they are faced with intimate care conflicts.

The structure and process are described below.

The model for facilitating the teaching of intimate care is the researchers' recommended framework for improving the provision of intimate care to diverse patients competently, confidently and comfortably. The model advocates for the facilitated teaching of intimate care to nursing students. They will be more empowered with intimate care knowledge and skills, and will be supported when experiencing difficulties in the execution or provision of intimate care.

The structural presentation of this model is displayed in Fig. 1. The model consists of four steps, i.e. step 1: intimate care conflict; step 2: intimate care facilitation; step 3: intimate care support; and step 4: therapeutic nurse-patient intimate relationship. These steps are discussed below.

\section{Step 1: Intimate care conflict}

Intimate care conflict is based on the diversity of nursing students and patients receiving nursing care. The participants in intimate care

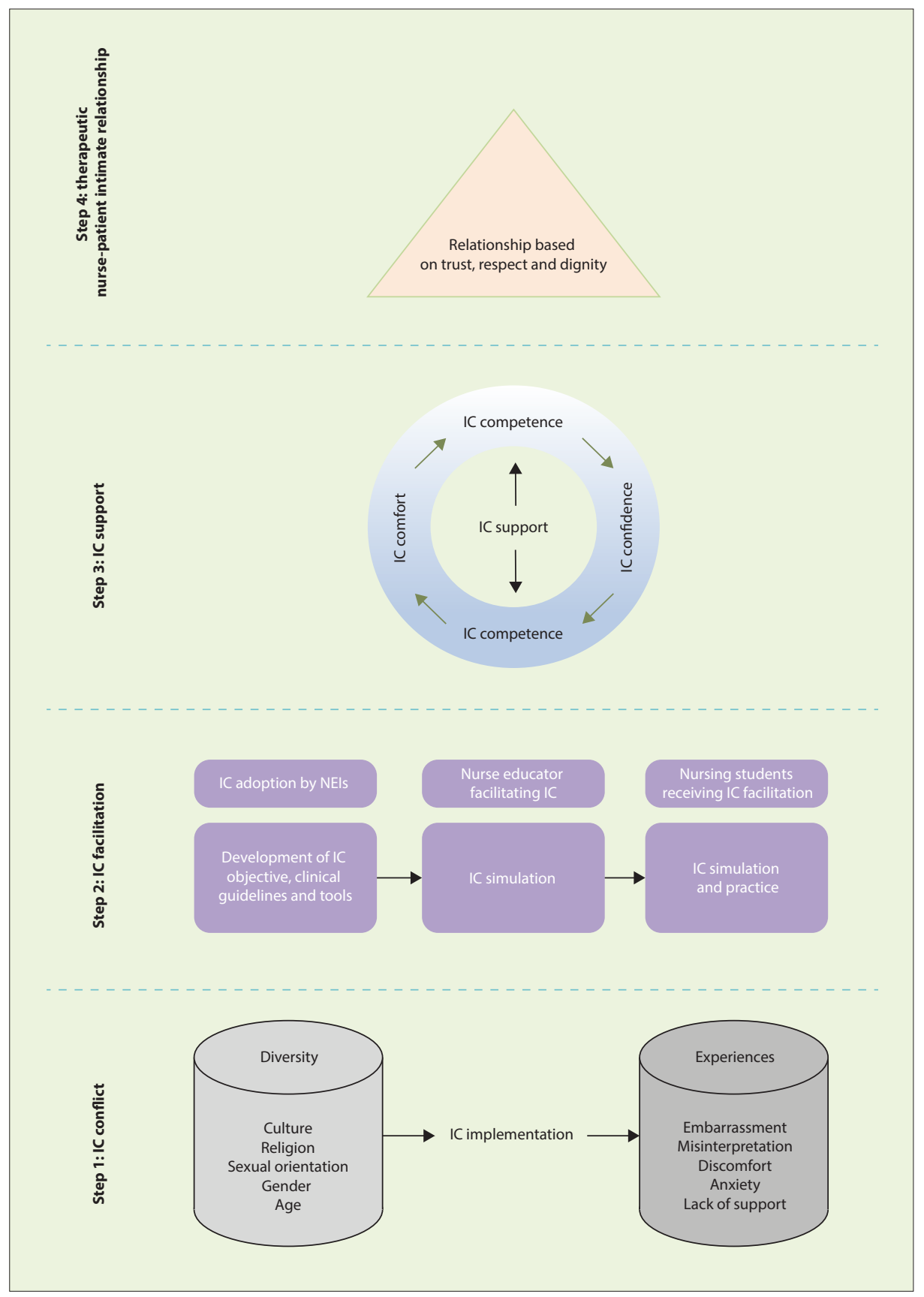

Fig. 1. Model for facilitating the teaching of intimate care. ${ }^{[18]}(I C=$ intimate care; $N E I=$ nursing education institution.) 
implementation are from diverse cultural and religious backgrounds, with different gender, age and sexual orientations. Nursing students bring with them sociocultural values and beliefs about touch and intimacy. For many, such practices occur within the safety of family and intimate relationships. ${ }^{[29]}$ The nursing students' background influences the beliefs and behaviours in caring for diverse patients. ${ }^{[30]}$ The intimate care conflict is assumed to be based on nursing students often not being well prepared to provide intimate care to diverse patients. ${ }^{[14]}$ When providing intimate care, many nursing students experience embarrassment and discomfort. There is also the possibility that intimate care could be misinterpreted as inappropriate or sexual by the patient. These experiences have led to intimate care conflict between nursing students and patients. ${ }^{[18]}$ During the execution of intimate care, nursing students may transgress their internalised social and cultural taboos. This contradiction may leave them feeling confused and vulnerable. ${ }^{[29]}$ Lack of intimate care knowledge, skills and support from NEIs and clinical facilities may lead to intimate care conflict.

\section{Step 2: Intimate care facilitation}

- NEIs adopting the intimate care concept: NEIs should acknowledge and adopt intimate care as a knowledge and skill that needs to be taught to nursing students. Intimate care should be included in the nursing curriculum - in theory and practice. Intimate care objectives/outcomes, procedural steps/ strategies and assessment tools should be developed and included in the nursing students' clinical procedure manual and clinical outcomes.

- Nurse educators facilitating the teaching of intimate care: in the study by O'Lynn and Kraustcheid, ${ }^{[8]}$ they show that intimate care instruction empowered male nursing students to feel more comfortable and maintain dignity, comfort and respect regarding the patient. Simulation is a strategy that can be used to facilitate the teaching of intimate care. Crossan and Mathew ${ }^{[7]}$ indicate that a humanistic form of simulation is needed to facilitate intimate care teaching. Mainey et al. ${ }^{[14]}$ used Mask-Ed simulation to facilitate intimate care education. This simulation approach is considered a humanistic form of simulation, as the educator becomes immersed in the teaching session by wearing a silicone mask and body parts, and is thus able to engage with students through the character of a patient. The students experienced both the physical and emotional aspects of providing safe intimate care, they developed confidence and felt more comfortable about providing such care. ${ }^{[4,14]}$

- Nurse educators delivering intimate care knowledge, skills and guidance to nursing students: nurse educators need to be intimate care competent, comfortable and confident. Nurse educators are trained in humanistic simulation of intimate care, such as using Mask-Ed simulation. The discussion of sociocultural and ethical issues related to intimate care and touch needs to be included. The training will assist them in dealing with their personal issues and experiences of intimate care.

- Nursing students receiving facilitated teaching in intimate care: nursing students are the recipients of intimate care knowledge, skills and guidance. They can discuss their diverse sociocultural, religious or other convictions in a safe environment. They participate in a humanistic simulation activity, where they experience the reality of intimate care. This simulation allows them to discover that the patient is at the centre of intimate care and is treated with respect and dignity.

\section{Step 3: Intimate care support}

Currently, when nursing students interact with strangers and their bodies during the execution of intimate care, they are often given very little or no support in this regard during their educational preparation. ${ }^{[7]}$ The third step assumes that the second step was implemented, i.e. that nursing students have received intimate care teaching, which was facilitated through discussion and humanistic simulation. They demonstrate that they have acquired the knowledge and skills required for the provision of intimate care and can be trusted to provide such care. Nursing students are now more competent, comfortable and confident in providing intimate care to patients of different genders, ages, cultures, religions and sexual orientation.

\section{Step 4: Therapeutic nurse-patient intimate relationships}

Mainey et al. ${ }^{[14]}$ define intimate care as the nurse-patient interaction, where the nurse enters the person's private zone, providing task-orientated care. The fourth step is based on the assumption that, if nursing students are knowledgeable, skilled and supported in the provision of intimate care, they will be better able to establish therapeutic nurse-patient intimate care relationships. Intimate care conflicts are reduced, and nursing students can negotiate and establish an intimate therapeutic relationship based on trust, respect and dignity. Moreover, patients are more likely to accept the provision of intimate care, without misinterpreting it as inappropriate or sexual. Both nurse and patient enter a trustful, respectful and dignified relationship.

\section{Ethical approval}

The paper forms part of a larger theory generative qualitative study approved by UNISA's Health Studies Research Ethics Committee (ref. no. HSHDC/496/2015).

\section{Study limitations}

This paper presents the steps used to develop a model for facilitating the teaching of intimate care. The model has not yet been implemented or operationalised at NEIs in SA. The researchers have presented the model to various stakeholders in NEIs in the public and private sectors. They are in the process of implementing and evaluating the model for clarity, consistency, simplicity, generality and accessibility.

\section{Conclusion and recommendations}

This article comprises a comprehensive description of a model for facilitating the teaching of intimate care, using the three steps of model development as supported by Chinn and Kramer ${ }^{[16]}$ and Dickoff et al ${ }^{[19]}$ A graphic representation of the model enhanced the structural clarity of the concepts within the model. The purpose of developing this model was to empower nursing students with intimate care knowledge and skills.

The model must be operationalised in nursing education, practice and research. Further research studies should be conducted to evaluate the effectiveness and feasibility of the model. It is envisaged that the developed model will empower nursing students to provide intimate care competently, comfortably and confidently. They will be able to establish nurse-patient intimate care relationships based on trust, respect and dignity. Research should also be done to understand the patients' experiences of intimate care. 
This will give a voice to the patients and add value to developing acceptable intimate care strategies in an African context.

\section{Declaration. None.}

Acknowledgements. We acknowledge the Gauteng Department of Health, selected NEIs for granting permission to conduct the study, and nurse educators and students who participated in the study.

Author contributions. The authors worked together on the manuscript. SS wrote the first draft and SMZ reviewed and corrected it before submission. SS and SMZ revised the manuscript according to the reviewers' comments. SS takes full responsibility for the study.

\section{Funding. None.}

Conflicts of interest. None.

1. Harding T, North N, Perkins R. Sexualizing men's touch: Male nurses and the use of intimate touch in clinica practice. Res Theory Nurs Pract 2008;22(2):88-102. https://doi.org/10.1891/1541-6577.22.2.88

. Lehn-Christiansen S, Holen M. Ambiguous socialisation into nursing: Discourses of intimate care. Nurse Educ Today 2019;75:1-5. https://doi.org/10.1016/j.nedt.2019.01.002

3. Pedrazza M, Minuzzo S, Berlanda S, Trifiletti E. Nurses' comfort with touch and workplace well-being. West J Nurs Res 2015;37(6):781-798. https://doi.org/10.1177/0193945914527356

4. Reid-Searl K, Mainey L, Bassett J, Dwyer T. Using simulation to prepare neophyte nursing students to delive intimate patient care. Collegian 2018;553:1-8. https://doi.org/10.1016/j.colegn.2018.08.001

5. Picco E, Santoro R, Garrino L. Dealing with the patient's body in nursing: Nurses' ambiguous experience in clinical practice. Nurs Inq 2010;17(1):39-46. https://doi.org/10.1111/j.1440-1800.2009.00481.x

6. Kirk TW. Beyond empathy: Clinical intimacy in nursing practice. Nurs Philos 2007:8(4):233-243. https://doi. 6. Kirk TW. Beyond empathy: Clinical intin
org/10.1111/.1466-769x.2007.00318.x

7. Crossan M, Mathew TK. Exploring sensitive boundaries in nursing education: Attitudes of undergraduate studen 7. Crossan M, Mathew TK. Exploring sensitive boundaries in nursing education: Attitudes of undergraduate studen
nurses providing intimate care to patients. Nurse Educ Pract 2013;13(4):317-322. https://doi.org/10.1016/j. nepr.2013.04.007

8. O'Lynn C, Krautscheid L. Evaluating the effects of intimate touch instruction: Facilitating professional and respectful touch by male nursing students. J Nurs Educ 2014;53(3):127-135. https://doi.org/10.3928/01484834-20140211-08 9. Morrell R, Jewkes R, Lindegger G. Hegemonic masculinity/masculinities in South Africa: Culture, power and gender politics. Men Masculin 2012;15(1):11-30. https://doi.org/10.1177\%2F1097184X12438001

10. Stanley D. Celluloid: A research study of male nurses in feature films. J Adv Nurs 2012;68(11):2526-2535. https:// doi.org/10.1111/j.1365-2648.2012.05952.x
11. Whiteside J, Butcher D. Not a job for a man': Factors in the use of touch by male nursing staff. Br J Nurs 2015;24(6):335-341. https://doi.org/10.12968/bjon.2015.24.6.335

12. Hollup O. The impact of gender, culture, and sexuality on Mauritian nursing: Nursing as a non-gendere occupational identiity or masculine field? Int J Nurs Stud 2014;51(5):752-760. https://doi.org/10.1016/. ijnurstu.2013.09.013

13. Metz T. Ubuntu as a moral theory and human rights in South Africa. Afr Hum Rights Law J 2011;1(2):532-359 https://hdl.handle.net/10520/EJC51951 (accessed 9 September 2020).

14. Mainey L, Dwyer T, Reid-Searl K, Bassett J. High-level realism in simulation: A catalyst for providing intimate care Clin Simul Nurs 2018;17:47-57. https://doi.org/10.1016/j.ecns.2017.12.001

15. Bednarz H, Schim S, Doorenbos A. Cultural diversity in nursing education: Perils, pitfalls, and pearls. J Nurs Educ 2010;49(5):253-260. https://doi.org/10.3928\%2F01484834-20100115-02

16. Chinn PL, Kramer MK. Integrated Theory and Knowledge Development in Nursing. 8th ed. St. Louis: Elsevier 2011.

17. Walker LO, Avant KC. Strategies for Theory Construction in Nursing. 5th ed. New Jersey: Pearson/Prentice Hall, 2011.

18. Shakwane S. A model for facilitating the teaching of intimate care to nursing students in South Africa. PhD thesis Pretoria: UNISA, 2017.

19. Dickoff J, James P, Wiedenbach E. Theory in a practice discipline. Practice-oriented research. Nurs Res 1968;17(6):415-435.

20. Regmi K. A review of teaching methods - lecturing and facilitation in higher education (HE): A summary of the published evidence. J Effect Teach 2012;12(3):61-76.

21. Mellish JM, Brink HIL, Paton F. Teaching and Learning the Practice of Nursing. Johannesburg: Heinemann, 2009. 22. Haavardsholm I Naden D. The concept of confidence: The nursés perception. Eur I Cancer Care 2009:18:483-491. https://doi.org/10.1111/j.1365-2354.2008.00993.x

23. Tilley DS. Competency in nursing: A concept analysis. J Contin Educ Nurs 2008;39(2):58-64. https://doi. org/10.3928/00220124-20080201-12

24. Cowan DT, Norman I, Coopamah VP. Competence in nursing practise: A controversial concept - a focused review of literature. Nurse Educ Today 2005;25(5):355-362. https://doi.org/10.1016/.nedt.2005.03.002

25. Nashwan AJ.An analysis of the concept in nursing education. 2015. https://www.researchgatenet/publication/281068340 (accessed 12 April 2017)

26. Kolcaba KY. A theory of holistic comfort. J Adv Nurs 1994:19:1178-1184. https://doi.org/10.1111/j.1365-2648.1994. tb01202

27. Nurses Association of New Brunswick. The therapeutic nurse-client relationship. 2011. www.nanb.nb.ca $>$ PDF $>$ PracticeStand (accessed 12 June 2015).

28. Prideaux A. Male nurses and the protection of female patient dignity. Art Sci Prof Iss 2010; 25(13):42-49. https://do org/10.7748/ns2010.12.25.13.42.c8121

29. Grant B, Giddings L, Beala JE. Vulnerable bodies competing discourse of intimate body care. J Nurs Educ 2005;44(11):498-504. https://doi.org/10.3928/01484834-20051101-05

30. Buthelezi F. Fakude LP, Martin P. Daniels F. Clinical learning experiences of male nursing students in a Bachelor of nursing programmes Strategies to overcome challenges. Curationis 2015:38(2):1-7. https://doi org $/ 10.4102$ curationis.v38i2.1517

Accepted 19 June 2020 